\section{Estudio in vitro de la relación entre el foramen apical y ápice anatómico}

\section{In vitro study of the relation between the apical foramen and anatomical apex}

\begin{abstract}
Resumen
Objetivo: Evaluar la relación entre la posición del foramen apical y el ápice anatómico en piezas dentarias uniradiculares. Materiales y método: Estudio in vitro que incluyó 71 piezas dentarias (incisivos, caninos y premolares) uniradiculares. Se evaluó la posición del foramen apical (vestibular, palatino/lingual, mesial y distal) así como la distancia desde el foramen apical hasta el vértice del ápice anatómico a través de un Vernier electrónico. Resultados: Treinta y dos piezas dentarias $(45,1 \%)$ coincidieron la ubicación entre el foramen apical y ápice anatómico; el 56,4\% de forámenes apicales se encontró a nivel vestibular y palatino/lingual. De las piezas dentarias donde se encontró la coincidencia, la mayor cantidad fue encontrada a nivel de los incisivos centrales superiores $(31,3 \%)$. La distancia entre la ubicación del foramen apical y el ápice anatómico fue de 0,36 $\pm 0,41$ mm. Conclusiones: El foramen apical se ubicó en mayor frecuencia a nivel vestibular y palatino/lingual. La coincidencia entre la posición del foramen apical y el vértice del ápice anatómico se encontró presente en un $45 \%$ de casos. La distancia promedio entre ambas estructuras anatómicas fue de 0,36 $\pm 0,41 \mathrm{~mm}$; siendo esta distancia mayor a nivel de premolares superiores.
\end{abstract}

Palabras clave: Anatomía; Ápice del diente; Endodoncia; Tratamiento del conducto radicular.

\begin{abstract}
Objective: To evaluate the relationship between the position of the apical foramen and the anatomic apex in uniradicular teeth. Materials and method: In this vitro study that included 71 teeth between incisors, canines and premolars, all of them were uniradicular. The position of the apical foramen (vestibular, palatal / lingual, mesial and distal) was evaluated as well as the distance from the apical foramen to the apex of the anatomic apex through an electronic Vernier. Results: In thirty-two dental pieces (45.1\%) coincided the location between the apical foramen and anatomical apex; in $40(56.4 \%)$ the apical foramen was found at vestibular and palatal / lingual levels, In teeth where there was concordance, the largest amount was found at the level of the upper central incisors $(31.3 \%)$. The distance between the position of the apical foramen and the anatomical apex was $0.36 \pm 0.41 \mathrm{~mm}$. Conclusions: The apical foramen is more frequent at the vestibular and palatine / lingual levels. The coincidence between the position of the apical foramen and the apex of the anatomical apex is present in $45 \%$ of cases. The average distance between both anatomical structures was $0.36 \pm 0.41 \mathrm{~mm}$; this distance being greater at the upper premolar.
\end{abstract}

Keywords: Anatomy; Endodontics; Root canal therapy; Tooth apex.
ISSN: $1560-9111$

\section{Artículo Original}

Piero Olivera-Rojas ${ }^{1, a}$, Wendy Lugo-Huertas ${ }^{1, a}$ Graciela Pumahualcca-García ${ }^{1, a}$, Rosa LaraVerástegui 1,a, Pamela Quispe-Romero 1,a, Yuri Castro Rodríguez $z^{2, b}$

${ }^{1}$ Sociedad Científica de Estudiantes de Odontología. Universidad Nacional Mayor de San Marcos. Lima, Perú.

${ }^{2}$ Facultad de Odontología. Universidad Nacional Mayor de San Marcos. Lima, Perú.

${ }^{a}$ Estudiante del pregrado.

${ }^{\text {b } C i r u j a n o ~ D e n t i s t a . ~}$

\section{Correspondencia:}

Piero Olivera Rojas

Correo electrónico: Piero.olivera@unmsm.edu. pe Facultad de Odontología, UNMSM. Calle Germán Amézaga 375. Lima 1, Perú.

\section{Coautores:}

Wendy Lugo-Huertas wendy.lugo@unmsm.edu.pe Graciela Pumahualcca-García graciela.pumahualcca@unmsm.edu.pe Rosa Lara-Verástegui rosa.lara1@unmsm.edu.pe Pamela Quispe-Romero pamela.quispe1@unmsm.edu.pe Yuri Castro Rodríguez yuricastro_16@hotmail.com

Conflicto de intereses: Los autores declaran no tener conflictos de interés.

Fuente de financiamiento: Autofinanciado.

Fecha de recepción: 07/05/17

Fecha de aceptación: 05/09/17

( ) Los autores. Este artículo es publicado por la revista Odontología Sanmarquina de la Facultad de Odontología, Universidad Nacional Mayor de San Marcos. Este es un artículo de acceso abierto, distribuido bajo los términos de la licencia Creative Commons Atribucion - No Comercia_Compartir Igual 4.0 Internacional. (http://creativecommons.org/licenses/by-nc-sa/4.0/) que permite el uso no comercial, distribución y reproducción en cualquier medio, siempre que la obra original sea debidamente citada. 


\section{Introducción}

El tratamiento de conductos representa una alternativa de gran importancia para conservar las piezas dentarias. La presencia de bacterias en el interior de los conductos radiculares desencadena procesos degenerativos que de acuerdo a su severidad causan diferentes patologías pulpares.

Una vez que se ha producido la invasión microbiana, el órgano dentino-pulpar reacciona a través de procesos inflamatorios y respuestas inmunitarias como mecanismos de defensa originando daños irreversibles en sus funciones fisiológicas ${ }^{1}$. Conocer la conformación anatómica de los conductos radiculares y anatomía interna de las piezas dentarias permite realizar tratamientos más conservadores e influir en el éxito del tratamiento de conductos ${ }^{2}$.

La porción apical de los dientes constituye un elemento clave en Endodoncia; principalmente la ubicación del foramen apical para la obtención de una adecuada longitud de trabajo durante la preparación biomecánica de los conductos radiculares. Existen algunos inconvenientes que limitan la ubicación del foramen apical, así por ejemplo en años anteriores se creía que la posición de foramen apical coincidía con el ápice anatómico ${ }^{3}$, siendo modificado este concepto por una discrepancia entre el foramen y el ápice principalmente a nivel de pacientes jóvenes y adultos ${ }^{3}$.

Se han planteado algunas técnicas para ubicar con precisión el foramen apical, de esta forma la clásica radiografía periapical ha sido reemplazada por localizadores apicales y radiovisiografía que facilitan la obtención de la longitud de trabajo ${ }^{4,5}$. La mayoría de autores acotan que el foramen apical se encuentra $1 \mathrm{~mm}$ coronal al ápice anatómico ${ }^{6-8}$; sin embargo, otros autores como Martos y cols. ${ }^{9}$ plantean que esta distancia es menor a $1 \mathrm{~mm}$, Marroquin y cols. ${ }^{10}$ acotan una distancia entre $0,86 \mathrm{~mm}$ y $1 \mathrm{~mm}$ en molares inferiores y superiores; y Blaskovic y cols. ${ }^{11}$ encontraron distancias de 0,99 mm. Las discrepancias aumentan cuando se analizan distintos tipos de piezas dentarias, ubicación del foramen, método de análisis, entre otros. En la presente investigación se evaluó la relación existente entre la posición del foramen apical y el ápice anatómico en piezas dentarias uniradiculares.

\section{Materiales y Método}

Se diseñó un estudio analítico in vitro que incluyó piezas dentarias extraídas en la Clínica de Pregrado de la Facultad de Odontología de la Universidad Nacional Mayor de San Marcos (Lima, Perú) durante el año 2017.

Se analizaron piezas dentarias uniradiculares (incisivos, caninos y premolares) superiores e inferiores, la cantidad de dientes utilizados fue determinado de forma no probabilística por criterio según el número de pacientes que fueron atendidos y que requerían tratamientos de exodoncia dentaria; se siguieron las consideraciones éticas del Informe de Helsinki con el uso de células y tejidos de origen humano en procesos de investigación. Cada paciente firmó la historia clínica y consentimiento informado, en el que permitía el uso científico de los dientes extraídos.

Los dientes a evaluar tuvieron que poseer ápices maduros, encontrarse recién extraídos y ser uniradiculares; se excluyeron dientes con ápices abiertos, dientes no permeables, con conductos calcificados, con reabsorción externa, fracturas radiculares y cualquier condición que impida la observación directa del tercio apical (hipercementosis, calcificaciones, reabsorciones, etc.)

Previo al análisis de las piezas dentarias, éstas fueron lavadas con suero fisiológico y alisadas en su porción radicular con curetas periodontales Gracey 1/2 y 3/4 (HuFriedy, EEUU) para eliminar residuos y/o tejidos que pudieron quedar luego de la exodoncia. Una vez limpiadas y seleccionadas las piezas dentarias se procedió a realizar la apertura cameral de cada diente. La apertura se realizó en aquellas piezas dentarias con porción coronaria intacta; se utilizó fresas redondas de carburo de tungsteno hasta encontrar los conductos radiculares. La localización de los conductos se realizó a través de exploradores y confirmada con limas endodónticas números 08 y 10 (K flexofile ${ }^{\circledast}$, EEUU). Las limas permitieron verificar la posición de los conductos radiculares así como localizar el foramen apical (Figura 1. A).

Una vez localizado el foramen apical se procedió a evaluar las siguientes variables: posición del foramen (considerado como vestibular, mesial, distal y palatino/ lingual), distancia del foramen apical al ápice anatómico
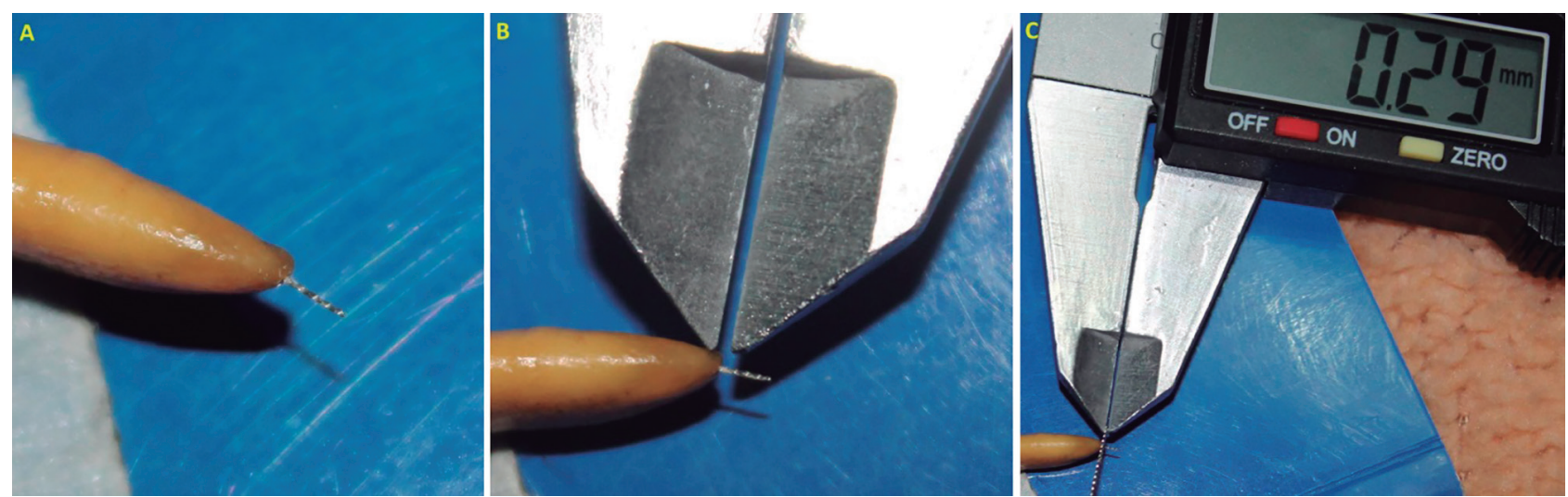

Figura 1. A. Localización del foramen apical. B. Distancia entre foramen apical y ápice anatómico. C. Registro de la medición con calibrador de precisión. 
(se trazó una línea recta paralela al eje de la raíz desde el punto más apical del foramen apical y una línea tangente en el punto más apical del ápice anatómico; la medición entre estas dos líneas fue realizada con un calibrador electrónico de precisión Vernier (Mitutoyo ${ }^{\circ}$, EEUU) (Figura 1. B y 1. C). En los casos que esta distancia fue de cero, se consideró como coincidencia entre el foramen apical y el ápice anatómico.

Para el análisis de datos descriptivo se utilizó distribución de frecuencias absolutas y relativas, se determinó la media, mediana y desviación estándar de la distancia en milímetros del forman apical al ápice anatómico. Para establecer diferencias entre las mediciones según tipo de diente se utilizó la prueba de ANOVA de un factor, mientras que para establecer una relación entre posición del foramen y pieza dentaria se utilizó un análisis de chi-cuadrado. Se aceptó un nivel de significancia de 0,05 para la refutación de la hipótesis nula.

\section{Resultados}

Fueron evaluadas 71 piezas dentarias; de las cuales el $29,6 \%$ fueron premolares inferiores, el $21,1 \%$ incisivos centrales superiores, $16,9 \%$ caninos superiores y $9,9 \%$ premolares superiores; en menor cantidad caninos inferiores $(7 \%)$, incisivos laterales superiores $(7 \%)$, incisivos centrales inferiores $(5,6 \%)$ e incisivos laterales inferiores $(2,8 \%)$.

Treinta y dos piezas dentarias $(45,1 \%)$ coincidieron la ubicación entre el foramen apical y ápice anatómico (Figura 1). De las 39 piezas restantes $(54,9 \%)$ el $56,4 \%$ de forámenes apicales se encontró a nivel vestibular y palatino/lingual (28,2\% para cada ubicación por igual), el $23,9 \%$ en posición mesial y en menor porcentaje en posición distal (19,7\%) (Tabla 1).

A nivel del incisivo central superior el $46,7 \%$ de forámenes se encontraron en posición palatina, en el incisivo lateral superior el $60 \%$ a nivel vestibular y a nivel canino superior el $50 \%$ en posición palatina ( $p>0,05)$ (Tabla 1$)$.

De las piezas dentarias donde se encontró coincidencia, la mayor cantidad fue encontrada a nivel de los incisivos centrales superiores $(31,3 \%)$, caninos superiores $(25 \%)$ $y$ premolares inferiores $(25 \%)(\mathrm{p}<0,05)$ (Figura 2$)$.

La distancia entre la ubicación del foramen apical y el ápice anatómico fue de $0,36 \pm 0,41 \mathrm{~mm}$ con un valor

Tabla 1. Posición del foramen apical según pieza dentaria.

\begin{tabular}{|c|c|c|c|c|c|c|c|c|c|}
\hline & $\begin{array}{l}\text { Central } \\
\text { Superior }\end{array}$ & $\begin{array}{c}\text { Lateral } \\
\text { Superior }\end{array}$ & $\begin{array}{l}\text { Canino } \\
\text { Superior }\end{array}$ & $\begin{array}{l}\text { Premolar } \\
\text { Superior }\end{array}$ & $\begin{array}{l}\text { Central } \\
\text { Inferior }\end{array}$ & $\begin{array}{l}\text { Lateral } \\
\text { Inferior }\end{array}$ & $\begin{array}{l}\text { Canino } \\
\text { Inferior }\end{array}$ & $\begin{array}{l}\text { Premolar } \\
\text { inferior }\end{array}$ & Total \\
\hline Vestibular & $5(25 \%)$ & $3(15 \%)$ & $2(10 \%)$ & $1(5 \%)$ & $1(5 \%)$ & $1(5 \%)$ & 0 & $7(35 \%$ & $20(28,2 \%)$ \\
\hline Mesial & $2(11,8 \%)$ & $1(5,9 \%)$ & $2(11,8 \%)$ & $1(5,9 \%)$ & $2(11,8 \%)$ & $1(5,9 \%)$ & 0 & $8(47,1 \%)$ & $17(23,9 \%)$ \\
\hline Distal & $1(7,1 \%)$ & 0 & $2(14,3 \%)$ & $4(28,6 \%)$ & $1(7,1 \%)$ & 0 & $2(14,3 \%)$ & $4(28,6 \%)$ & $14(19,7 \%)$ \\
\hline Palatino/Lingual & $7(35 \%)$ & $1(5 \%)$ & $6(30 \%)$ & $1(5 \%)$ & 0 & 0 & $3(15 \%)$ & $2(10 \%)$ & $20(28,2 \%)$ \\
\hline Total & $15(21,1 \%)$ & $5(7 \%)$ & $12(16,9 \%)$ & $7(9,9 \%)$ & $4(5,6 \%)$ & $2(2,8 \%)$ & $5(7 \%)$ & $21(29,6 \%)$ & $71(100 \%)$ \\
\hline
\end{tabular}

$\mathrm{P}=0,088 \quad \mathrm{X}^{2}=30,213 \quad \mathrm{gl}=21$

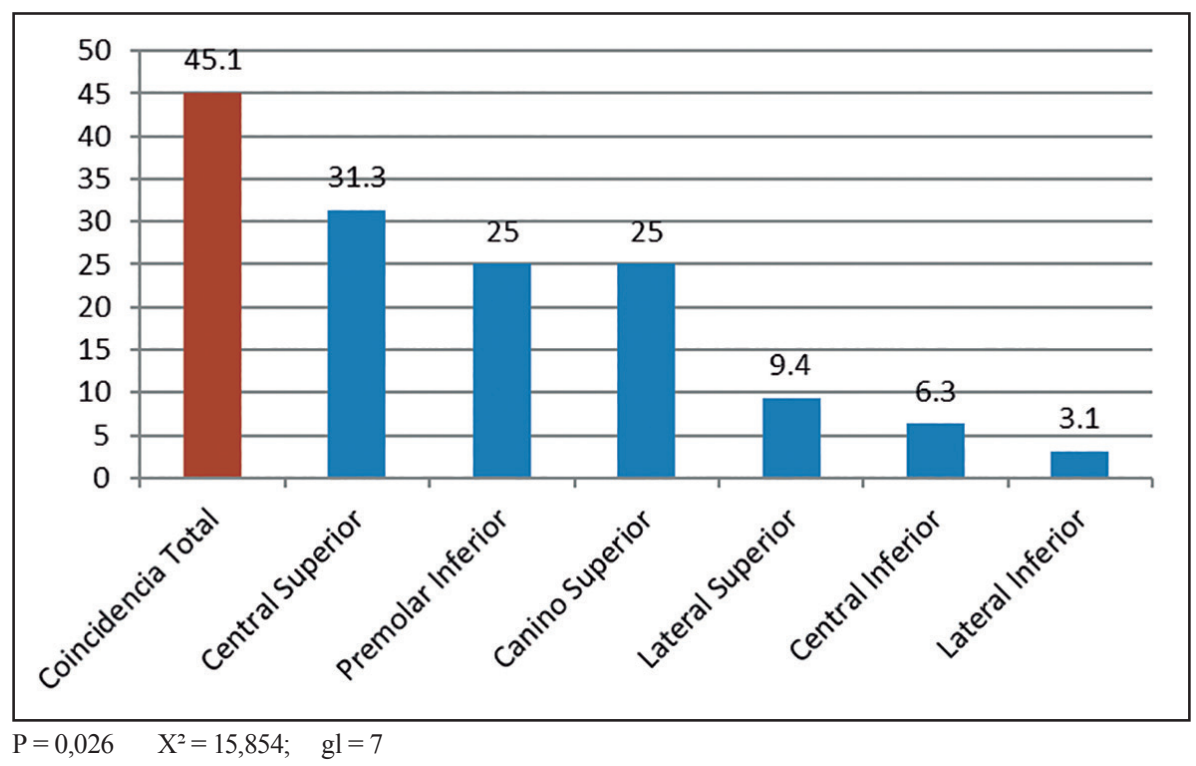

Figura 2. Porcentaje de coincidencia entre foramen apical y ápice anatómico según pieza dentaria 
Tabla 2. Promedios de la distancia entre foramen apical y ápice anatómico según pieza dentaria.

\begin{tabular}{|c|c|c|c|c|c|c|c|c|}
\hline Central Superior & $\begin{array}{l}\text { Lateral } \\
\text { Superior }\end{array}$ & $\begin{array}{l}\text { Canino } \\
\text { Superior }\end{array}$ & $\begin{array}{l}\text { Premolar } \\
\text { Superior }\end{array}$ & $\begin{array}{l}\text { Central } \\
\text { Inferior }\end{array}$ & $\begin{array}{l}\text { Lateral } \\
\text { Inferior }\end{array}$ & $\begin{array}{l}\text { Canino } \\
\text { Inferior }\end{array}$ & $\begin{array}{l}\text { Premolar } \\
\text { inferior }\end{array}$ & Total \\
\hline $\begin{array}{c}0,2 \pm 0,29^{\mathrm{ab}} \mathrm{mm} \\
(0-0,68 \mathrm{~mm})\end{array}$ & $\begin{array}{c}0,22 \pm 0,31^{\mathrm{ab}} \\
(0-0,65)\end{array}$ & $\begin{array}{c}0,09 \pm 0,15^{\mathrm{a}} \\
(0-0,39)\end{array}$ & $\begin{array}{c}0,71 \pm 0,38^{\mathrm{bc}} \\
(0,18-1,14)\end{array}$ & $\begin{array}{c}0,26 \pm 0,3^{\mathrm{ab}} \\
(0-0,53)\end{array}$ & $\begin{array}{c}0,28 \pm 0,33^{\mathrm{ab}} \\
(0-0,56)\end{array}$ & $\begin{array}{l}0,6 \pm 0,22^{\mathrm{ab}} \\
(0,39-0,83)\end{array}$ & $\begin{array}{c}0,52 \pm 0,53^{\text {bc }} \\
(0-1,78)\end{array}$ & $\begin{array}{c}0,36 \pm 0,41 \\
(0-1,78)\end{array}$ \\
\hline
\end{tabular}

$* \mathrm{P}<0,05$ ANOVA, Post Hoc de Tukey.

$\uparrow$ Letras diferentes indican diferencias estadísticamente significativas.

máximo de $1,78 \mathrm{~mm}$; siendo la distancia mayor a nivel de premolares superiores $(0,71 \pm 0,38 \mathrm{~mm})$ y menor para el canino superior $(0,09 \pm 0,31 \mathrm{~mm})(\mathrm{p}<0,05)$ (Tabla 2).

El intervalo de confianza de la distancia entre el foramen apical y ápice anatómico al 95\% fue más amplio para el incisivo lateral inferior $(-3,27-3,83)$ y menor para el canino superior $(-0,006-1,86)$ (Figura 3$)$.

\section{Discusión}

Para obtener un tratamiento endodóntico exitoso es necesario del conocimiento de la morfología normal y de las variaciones anatómicas del sistema de conductos radiculares ${ }^{12}$. En la presente investigación se evalúo el foramen apical y la relación con el ápice anatómico. El foramen apical constituye la circunferencia en forma de cráter o túnel que diferencia la terminación del conducto cementario de la superficie exterior de la raíz ${ }^{13}$; mientras que el ápice anatómico implica el extremo de la raíz, este ápice comprende los 2-3 mm finales de la raíz y su punto más extremo es el vértice radicular ${ }^{13}$.

El conocimiento de la ubicación del foramen apical repercute en la longitud de trabajo que se utilizará durante la preparación biomecánica. La comprensión de la anatomía de los conductos y ápice radicular permite realizar un tratamiento más conservador así como reducir los factores que pueden llevar a provocar complicaciones clínicas ${ }^{12}$. El presente estudio encontró una mayor frecuencia de forámenes apicales en posición vestibular y palatino/lingual; resultados similares a De Vasconcelos y cols. quienes encontraron una mayor presencia a nivel vestibular ${ }^{14}$. La mayor frecuencia del foramen en estas ubicaciones limita el análisis radiográfico durante el tratamiento de conductos; esto debido a que la ubicación del foramen apical en radiografías es alterada por la superposición de tejidos ${ }^{15}$. La ubicación vestibular o palatina no sería perceptible a través de radiografías, lo que dificultaría la localización del foramen apical. La localización mesial y distal obtuvo las menores frecuencias en nuestra investigación; ubicar el foramen en distal o mesial puede ser visible en la radiografía periapical al realizar la evaluación de longitud de trabajo, esto evitaría accidentes clínicos al permitir precurvar las limas para lograr la permeabilidad ${ }^{12}$.

Del total de piezas dentarias evaluadas se encontró una relación entre foramen apical y ápice anatómico en el $45 \%$ de casos. Ramos y cols. ${ }^{2}$ mencionan una relación

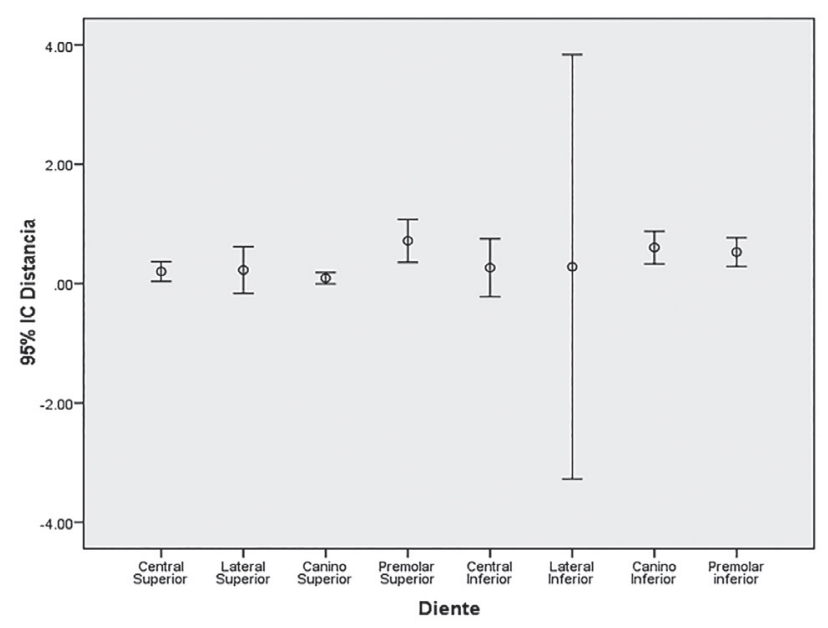

Figura 3. Intervalos de confianza de la distancia entre foramen apical y ápice anatómico según pieza dentaria.

entre la posición del ápice anatómico y el foramen apical en el $40 \%$ de casos; Kuttler ${ }^{16}$ una relación del 32\%; mientras que otro estudio menciona una discrepancia de $69,3 \%$ en dientes anteriores y de $50 \%$ en posteriores ${ }^{17}$. Mizufani y cols. ${ }^{18}$ encontraron una coincidencia del $16,7 \%$ de los incisivos centrales y caninos superiores y un $6,7 \%$ en los incisivos laterales superiores entre el vértice apical y el foramen apical. De las distintas investigaciones 16-18 se infiere que dependiendo del tipo de pieza dentaria el foramen apical suele ubicarse a cierta distancia del vértice del ápice anatómico; esta distancia sería considerada como un factor anatómico influyente para el tratamiento de conductos y la instrumentación mecánica.

De los distintos estudios, algunos mencionan que la distancia del vértice del ápice anatómico al foramen apical es de $0,43 \pm 0,33 \mathrm{~mm}$, Dumer y cols. ${ }^{19}$ encuentran una distancia de 0,89 mm; Melius y cols. ${ }^{20}$ en radiografías convencionales encontraron que la distancia de foramen y el ápice fue de 0,494 mm; mientras que en radiografías digitales de $0,594 \mathrm{~mm}$. Asimismo, Marroquin y cols. ${ }^{10}$ encontró distancias de $0,86 \mathrm{~mm}$ y $1 \mathrm{~mm}$ en molares inferiores y superiores. Finalmente, Blaskovic y cols. ${ }^{11}$ encontraron distancias de $0,99 \mathrm{~mm}$. Los resultados de la investigación $(0,36 \pm 0,41 \mathrm{~mm})$ concuerdan con la mayoría de estudios mencionados y se asemeja a lo planteado por los autores, que el foramen apical se encuentra en promedio a $1 \mathrm{~mm}$ del vértice del ápice anatómico; 
esta consideración debe ser valorada al momento de la exploración de la longitud de trabajo y el manejo de las limas para evitar complicaciones como sobre instrumentaciones, dolor y/o extravasación de detritus hacia la zona periapical. Se recomienda continuar con la línea de investigación planteada, aumentar la cantidad de piezas dentarias a analizar, incluir a la evaluación piezas multiradiculares así como un análisis de los forámenes accesorios y su relación con el ápice anatómico.

\section{Conclusiones}

El foramen apical se ubica con mayor frecuencia a nivel vestibular y palatino/lingual en incisivos, caninos y premolares uniradiculares. La coincidencia entre la posición del foramen apical y el vértice del ápice anatómico se encuentra presente en un $45 \%$ de casos. La distancia promedio entre el foramen apical y ápice anatómico es de 0,36 $\pm 0,41 \mathrm{~mm}$; siendo esta distancia mayor a nivel de premolares superiores.

\section{Referencias bibliográficas}

1. Hargreaves KM, Cohen S. Vías de la Pulpa. 10a ed. Madrid: Elsevier; 2011.

2. Ramos MJ, Gómez AI, Ortiz PV, Díaz CJ. Relación entre foramen apical, ápice anatómico y ápice radiográfico en premolares maxilares. Rev Cub Estomatol. 2015;52(1):11-20.

3. Ghasemi N, Rahimi S, Shahi S, Samiei M, Frough-Reyhani M, Ranjkesh B. A review on root anatomy and canal configuration of the maxillary second molars. Iran Endod J. 2017;12(1):1-9. Doi: 10.22037/iej.2017.01.

4. ElAyouti A, Dima E, Lost C. A tactile method for canal length determination in teeth with open apices. Int Endod J. 2009;42(12):1090-5.

5. Ghaemmaghami S, Eberle J, Duperon D. Evaluation of the Root ZX apex locator in primary teeth. Pediatr Dent. 2008;30(6):496-8.

6. Saghiri MA, Asgar K, Boukani KK, Lotfi M, Aghili H, Delvarani A, et al. A new approach for locating the minor apical foramen using an artificial neural network. Int Endod J. 2012;45(3):257-65.

7. Gonzalez-Sanchez JA, Duran-Sindreu F, de Noe S, Mercade M, Roig M. Centring ability and apical transportation after overinstrumentation with ProTaper Universal and ProFile Vortex instruments. Int Endod J. 2012;45(6):542-51.

8. Nica L, Grigorie M, Rusu D, Anghel MM, Didilescu A, Stratul SI. Computer-assisted photomicrographic evaluation of root canal morphology after removal of the filling material during retreatment. Rom J Morphol Embryol. 2011;52(1 Suppl):443-8.

9. Martos J, Ferrer-Luque CM, Gonzalez-Rodriguez MP, Castro LA. Topographical evaluation of the major api- cal foramen in permanent human teeth. Int Endod J. 2009;42(4):329-34.

10. Marroquin BB, El-Sayed MA, Willershausen-Zonnchen B. Morphology of the physiological foramen: I. Maxillary and mandibular molars. J Endod. 2004;30(5):321-8.

11. Blaskovic V, Maricic B, Sutalo J. Asymmetry of the root canal foramen. Int Endod J. 1992;25(3):158-64.

12. Doussoulin GL, Galleguillos VC, Galvez BC. Número y disposición de los forámenes apicales en la superficie radicular de primeros premolares superiores. Int J Odontostomat. 2016;10(3):419-24.

13. Cohen S, Burns R. Las vías de la pulpa. 7a ed. San Francisco: Harcourt; 2008.

14. de Vasconcelos BC, do Vale TM, de Menezes AS, Pinheiro-Junior EC, Vivacqua-Gomes N, Bernardes RA, et al. An ex vivo comparison of root canal length determination by three electronic apex locators at positions short of the apical foramen. Oral Surg Oral Med Oral Pathol Oral Radiol Endod. 2010;110(2):57-61.

15. Shalini R, RaviVarman C, Manoranjitham R, Veeramuthu M. Morphometric study on mandibular foramen and incidence of accessory mandibular foramen in mandibles of south Indian population and its clinical implications in inferior alveolar nerve block. Anat Cell Biol. 2016; 49(4):241-248. Doi: 10.5115/acb.2016.49.4.241.

16. Kuttler Y. Microscopic investigation of root apices. J Am Dent Assoc. 1955;50:544-52.

17. Versiani MA, Ahmed HM, Sousa-Neto MD, De-Deus G, Dummer PM. Unusual deviation of the main foramen from the root apex. Braz Dent J. 2016;27(5):589-591. Doi: 10.1590/0103-6440201601106.

18. Mizutani T, Okuo W, Nakamura H. Anatomical study of the root apex in the maxillary anterior teeth. J Endod. 1992;18:344-7.

19. Dummer PM, McGinn J, Rees D. The position and topography of the apical canal constriction and apical foramen. Int Endod J. 1984;17:192-8.

20. Melius B, Jiang J, Zhu Q. Measurement of the distance between the minor foramen and the anatomic apex by digital and conventional radiography. J Endod. 2002;28:125-6. 